\title{
On the particle-hole symmetry of the fermionic spinless Hubbard model in $D=1$
}

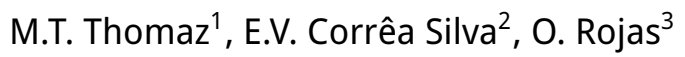 \\ ${ }^{1}$ Instituto de Física, Universidade Federal Fluminense, Av. Gal. Milton Tavares de Souza s/n ${ }^{\circ}$, CEP 24210-346, \\ Niterói-RJ, Brazil \\ 2 Departamento de Matemática, Física e Computação, Faculdade de Tecnologia, Universidade do Estado do Rio \\ de Janeiro, Rodovia Presidente Dutra km $298 \mathrm{~s} / \mathrm{n}^{0}$, Pólo Industrial, CEP 27537-000, Resende-RJ, Brazil \\ 3 Departamento de Ciências Exatas, Universidade Federal de Lavras, Caixa Postal 3037, CEP 37200-000, \\ Lavras-MG, Brazil
}

Received January 10, 2014, in final form February 25, 2014

\begin{abstract}
We revisit the particle-hole symmetry of the one-dimensional $(D=1)$ fermionic spinless Hubbard model, associating that symmetry to the invariance of the Helmholtz free energy of the one-dimensional spin-1/2 XXZ Heisenberg model, under reversal of the longitudinal magnetic field and at any finite temperature. Upon comparing two regimes of that chain model so that the number of particles in one regime equals the number of holes in the other, one finds that, in general, their thermodynamics is similar, but not identical: both models share the specific heat and entropy functions, but not the internal energy per site, the first-neighbor correlation functions, and the number of particles per site. Due to that symmetry, the difference between the first-neighbor correlation functions is proportional to the $z$-component of magnetization of the $X X Z$ Heisenberg model. The results presented in this paper are valid for any value of the interaction strength parameter $V$, which describes the attractive/null/repulsive interaction of neighboring fermions.
\end{abstract}

Key words: quantum statistical mechanics, strongly correlated electron system, spin chain models

PACS: 05.30.Fk, 71.27.+a, 75.10.Pq

The one-band Hubbard model [1,2] partially describes quantum magnetic phenomena; the complexity of real materials, however, imposes severe limitations on the direct comparison of experimental and theoretical results. It is not always clear which missing terms should be included in the fermionic Hamiltonian to account for the diversity of phenomena in a strongly correlated electron system.

The development of optical lattices over the last two decades has made the experimental simulation of chain models possible. The three-dimensional Hubbard model at low temperatures has been simulated by a fermionic quantum gas trapped in an optical lattice [3, 4]. A review of the simulation of the FermiHubbard model with fermionic atoms in optical lattices can be found in [5]. The simulation of a onedimensional spin-1/2 Ising model by a degenerate Bose gas of rubidium atoms confined in an optical lattice can be found in [6]. The simplest one-dimensional fermionic model is the fermionic spinless Hubbard model, the generalizations of which have been applied to the description of Verwey metal-insulator transitions and charge-ordering phenomena of the $\mathrm{Fe}_{3} \mathrm{O}_{4}, \mathrm{Ti}_{4} \mathrm{O}_{7}, \mathrm{LiV}_{2} \mathrm{O}_{4}$ and other $d$-metal compounds [7, 8].

In this paper we revisit the consequences of the particle-hole symmetry on the thermodynamics of the one-dimensional fermionic spinless Hubbard model in the whole range of temperatures, by mapping it into the exactly solvable $D=1$ spin-1/2XXZ Heisenberg model. Appendix A shows the $\beta$-expansion of the Helmholtz free energy (HFE) of this model, up to the order $\beta^{6}$ [9].

The spinless fermionic Hubbard model in $D=1$ is a very simple anti-commutative model the Hamiltonian of which is [10]:

$$
\mathbf{H}(t, V, \mu)=t \sum_{i=1}^{N}\left(\mathbf{c}_{i}^{\dagger} \mathbf{c}_{i+1}+\mathbf{c}_{i+1}^{\dagger} \mathbf{c}_{i}\right)+V \sum_{i=1}^{N} \mathbf{n}_{i} \mathbf{n}_{i+1}-\mu \sum_{i=1}^{N} \mathbf{n}_{i},
$$


in which $\left(\mathbf{c}_{i}, \mathbf{c}_{i}^{\dagger}\right)$, with $i \in\{1,2, \ldots, N\}$, are fermionic annihilation and creation operators, respectively, and $N$ is the number of sites in the chain. These operators satisfy anticommutation relations, $\left\{\mathbf{c}_{i}, \mathbf{c}_{j}^{\dagger}\right\}=\delta_{i j} \mathbb{1}_{i}$ and $\left\{\mathbf{c}_{i}, \mathbf{c}_{j}\right\}=0$, in which $t$ is the hopping integral, $V$ is the strength of the repulsion $(V>0)$ or attraction $(V<0)$ between first-neighbour fermions, $\mu$ is the chemical potential and $\mathbf{n}_{i}=\mathbf{c}_{i}^{\dagger} \mathbf{c}_{i}$ is the operator number of fermions at the $i^{\text {th }}$ site of the chain.

Sznajd and Becker [10] have shown that the Hamiltonian (1) has a particle-hole symmetry; consequently, the HFE of this model, $W(t, V, \mu ; \beta)$, satisfies the relation

$$
W(t, V, \mu ; \beta)=W(t, V,-\mu+2 V ; \beta)-(\mu-V),
$$

in which $\beta=\frac{1}{k T}, k$ is the Boltzmann's constant and $T$ is the absolute temperature in Kelvin. The relation (2) is valid for any values (positive, null or negative) of $V$ and $\mu$. Equation (2) provides the condition for having the same number of particles and holes at the same potential $V$ but at distinct chemical potentials,

$$
\left\langle\mathbf{n}_{i}\right\rangle(t, V, \mu ; T)=1-\left\langle\mathbf{n}_{i}\right\rangle(t, V,-\mu+2 V ; T),
$$

in which $\left\langle\mathbf{n}_{i}\right\rangle$ is the average number of fermionic particles at each site of the chain at temperature $T$.

Haldane [11] showed the equivalence of the model (1) and the spin-1/2 XXZ Heisenberg model in $D=1$. More recently, Sznajd and Becker [10] also used the inverse Wigner-Jordan transformation to show that the Hamiltonian (1) is mapped onto the Hamiltonian of the one-dimensional spin-1/2 $X X Z$ Heisenberg model with a longitudinal magnetic field,

$$
\mathbf{H}_{S=1 / 2}(J, \Delta, h)=\sum_{i=1}^{N}\left[J\left(\mathbf{S}_{i}^{x} \mathbf{S}_{i+1}^{x}+\mathbf{S}_{i}^{y} \mathbf{S}_{i+1}^{y}+\Delta \mathbf{S}_{i}^{z} \mathbf{S}_{i+1}^{z}\right)-h \mathbf{S}_{i}^{z}\right]
$$

in which $\mathbf{S}^{l}=\frac{\sigma^{l}}{2}, l \in\{x, y, z\}$, and $\sigma^{l}$ are the Pauli matrices. The norm of the spin operator $\overrightarrow{\mathbf{S}}$ is $\|\overrightarrow{\mathbf{S}}\|=\frac{\sqrt{3}}{2}$.

The Hamiltonians (1) and (4) are related by

$$
\mathbf{H}(t, V, \mu)=\mathbf{H}_{S=1 / 2}(J=2 t, \Delta=V /(2 t), h=\mu-V)-N\left(\frac{J \Delta}{4}+\frac{h}{2}\right) \mathbb{1}
$$

and $\mathbb{1}$ is the identity operator of the chain. This relation shows a constant shift between the energy spectrum of these two models.

Let $W_{S=1 / 2}(J, \Delta, h ; \beta)$ be the HFE associated to the Hamiltonian (4) of the $D=1$ spin-1/2XXZ Heisenberg model. A direct consequence of (5) is that

$$
W(t, V, \mu ; \beta)=W_{S=1 / 2}(J=2 t, \Delta=V /(2 t), h=\mu-V ; \beta)+\left(\frac{V}{4}-\frac{\mu}{2}\right) .
$$

At finite temperature ( $T \neq 0$ ), the HFE of the one-dimensional $S=1 / 2 X X Z$ Heisenberg model is an even function of the longitudinal magnetic field $h$,

$$
W_{S=1 / 2}(J, \Delta,-h ; T)=W_{S=1 / 2}(J, \Delta, h ; T) .
$$

Such invariance of $W_{S=1 / 2}$ comes from the symmetry of the Hamiltonian (4) upon reversal of the external magnetic field, $h \rightarrow-h$, and of the spin operators, $\overrightarrow{\mathbf{S}}_{i} \rightarrow-\overrightarrow{\mathbf{S}}_{i}$, in which $i \in\{1,2, \cdots N\}$.

Consider, for a given magnetic field $h$ and a fixed value (positive, null or negative) of $V$, the chemical potential $\mu$ so that $h=\mu-V$. For a reversed magnetic field, the corresponding chemical potential $\mu_{2}$ for which $-h=\mu_{2}-V$ is

$$
\mu_{2}=-\mu+2 V
$$

The identity (7) and the condition (8) recover the result (2) satisfied by the HFE of the spinless Hubbard model for any values of $V$ and $\mu$. Notice that in the half-filling condition $(\mu=V)$, we have $\mu_{2}=\mu$, and there is no visible consequence of the symmetry (7).

We point out that the quantity $-\mu+2 \mathrm{~V}$, which appears on the r.h.s. of (8), also appears as an argument of $W$ (the HFE of $D=1$ spinless fermionic Hubbard model) in the r.h.s. of (2), which in its turn comes 
from the particle-hole symmetry of the Hamiltonian (1). On the other hand, (7) comes from the fact that the HFE of the $D=1$ spin-1/2XXZ Heisenberg model is insensitive to a reversal of the longitudinal magnetic field.

Equation (3) can be interpreted as follows: the number of particles in the chain under a potential $V$ and a chemical potential $\mu$ equals the number of holes in the chain under the same potential $V$ and a chemical potential $\mu_{2}$ given by (8). Those configurations correspond to distinct distributions of the fermionic particles in the chain, and certainly have some different thermodynamic functions at temperature $T$. In what follows, we explore the consequences of the equality (7) in the thermodynamic functions of those two configurations.

The specific heat $C$ and the entropy $S$, both per site, are related to the HFE of the model (1) by $C(t, V, \mu ; \beta)=-\beta^{2} \frac{\partial^{2}}{\partial \beta^{2}}[\beta W(t, V, \mu ; \beta)]$ and $\frac{S}{k}=\beta^{2} \frac{\partial}{\partial \beta} W(t, V, \mu ; \beta)$, respectively. Due to equation $(6)$ we obtain

$$
C(t, V, \mu ; T)=C(t, V,-\mu+2 V ; T)
$$

and

$$
S(t, V, \mu ; T)=S(t, V,-\mu+2 V ; T) .
$$

Both $(9 \mathrm{a})$ and $(9 \mathrm{~b})$ are valid in the whole range of temperatures $T>0$. This can be verified at each order of the $\beta$-expansion of the thermodynamic functions derived from the expansion (16) of the HFE of the model, shown in appendix A.

However, not all thermodynamic functions of the model (1) are identical for the chemical potentials $\mu$ and $\mu_{2}$, at the same potential $V$. The internal energy per site $\varepsilon(t, V, \mu ; \beta)=\frac{\partial}{\partial \beta}[\beta W(t, V, \mu ; \beta)]$ distinguishes the distributions of the fermionic particles in the chain:

$$
\varepsilon\left(t, V, \mu_{2} ; \beta\right)=-V+\mu+\varepsilon(t, V, \mu ; \beta) .
$$

Notice that the difference of internal energies per site does not depend on the temperature. This equality is valid for any temperature $T>0$ and it is verified at each order of the expansion in $\beta$ for this thermodynamic function, obtained from (16).

In the spin-1/2 Heisenberg model (4), the parallel and anti-parallel configurations of spin with respect to the external magnetic field can be distinguished, for instance, by the average value of the $z$-component of the spin operator $\mathbf{S}_{i}^{z}$ at a site and the correlation function of odd powers of such operators. In terms of fermionic operators, we have $\mathbf{S}_{i}^{z}=\mathbf{n}_{i}-\frac{1}{2} \mathbb{1}_{i}$, in which $\mathbb{1}_{i}$ is the identity operator at the $i$-th site.

For the spinless fermionic Hubbard model, the first-neighbor correlation function $\mathbf{G}_{i, i+1}(t, V, \mu ; T) \equiv$ $\left\langle\mathbf{n}_{i} \mathbf{n}_{i+1}\right\rangle$ also relates configurations in which the number of particles in one equals the number of holes in the other, for two values of the chemical potential, $\mu$ and $\mu_{2}$.

The two-point correlation function $\mathbf{G}_{i, i+1}$ is related to the HFE by

$$
\mathbf{G}_{i, i+1}(t, V, \mu ; T)=\frac{\partial W(t, V, \mu ; T)}{\partial V} .
$$

From relation (6), the symmetry condition (7) and the definition of the $z$-component of the magnetization of the $D=1$ spin-1/2XXZ Heisenberg model,

$$
\begin{aligned}
M_{z}^{S=1 / 2}(J, \Delta, h ; T) & =-\frac{\partial W_{S=1 / 2}(J, \Delta, h ; T)}{\partial h} \\
& =\left\langle\mathbf{S}_{i}^{z}\right\rangle(J, \Delta, h ; T),
\end{aligned}
$$

in which $i \in\{1,2, \cdots, N\}$ and $\left\langle\mathbf{S}_{i}^{z}\right\rangle(J, \Delta, h ; T)$ is the mean value of the $z$-component of the spin- $1 / 2$ operator at the $i^{\text {th }}$ site of the chain and at temperature $T$, we obtain

$$
\mathbf{G}_{i, i+1}\left(t, V, \mu_{2} ; T\right)-\mathbf{G}_{i, i+1}(t, V, \mu ; T)=-2 M_{z}^{S=1 / 2}(J, \Delta, h ; T),
$$

where on its r.h.s. we have the $z$-component of the magnetization $M_{z}^{S=1 / 2}$ in the presence of a longitudinal magnetic field. (Notice that $M_{z}$ is a one-point function of the model, whereas $\mathbf{G}_{i, i+1}$ is a two-point 
function.) Equation (13) is valid for each order of the $\beta$-expansion of the function $\mathbf{G}_{i, i+1}(t, V, \mu ; \beta)$, derived from the expansion (16).

As a consequence of the symmetry in equation (7), the magnetization $M_{z}^{S=1 / 2}$ is an odd function of the magnetic field $h$,

$$
M_{z}^{S=1 / 2}(J, \Delta,-h ; T)=-M_{z}^{S=1 / 2}(J, \Delta, h ; T) .
$$

By writing equation 14 in terms of fermionic operators, $\mathbf{S}_{i}^{z}=\mathbf{n}_{i}-\frac{1_{i}}{2}$, one obtains

$$
\left\langle\mathbf{n}_{i}\right\rangle(t, V, \mu ; T)=1-\left\langle\mathbf{n}_{i}\right\rangle(t, V,-\mu+2 V ; T),
$$

thus, recovering equation (3).

In summary, we have verified that the particle-hole symmetry of the one-dimensional spinless fermionic Hubbard model (1) is associated to the invariance of the HFE of the $D=1$ spin-1/2 XXZ Heisenberg model (4) with respect to a reversal of the longitudinal external magnetic field.

The thermodynamics of the chain off the half-filling condition $(\mu \neq V)$ with chemical potentials $\mu$ and $\mu_{2}$, under the same potential $V$, are not identical; rather, some thermodynamic functions permit their distinction. Although the number of fermionic particles in the chain differ for $\mu$ and $\mu_{2}$, we obtain unexpected results, expressed in (9a) and (9b), where both configurations exhibit the same specific heat and entropy per site at any finite temperature $T$ and at any value of $V$. Distinction arises from other thermodynamic functions of the chain, though: the values of the internal energy per site of these two distributions of particles in the chain differ by a constant that is independent of the temperature; and the difference of their first-neighbour correlation functions is a one-point function proportional to the $z$-component of magnetization per site of the spin-1/2 model (4).

The equality of the number of particles in the chain for the chemical potential $\mu$ and the number of holes in the chain for the chemical potential $\mu_{2}$ is a consequence of the odd parity of magnetization $M_{z}^{S=1 / 2}(J, \Delta, h ; \beta)$ under reversal of the magnetic field $h \rightarrow-h$, for any temperature.

The results presented here are valid for any value of $V$ (negative, null or positive) and any value of temperature $T>0$, verified at each order of the $\beta$-expansion of the respective thermodynamic function. These results are also valid at very low temperatures and could be checked in an optical lattice simulation of the one-dimensional fermionic spinless Hubbard model.

\section{Acknowledgements}

E.V. Corrêa Silva thanks CNPq (Fellowship CNPq, Brazil, Proc. No. 303876/2010-7) for partial financial support. O. Rojas thanks CNPq and FAPEMIG for partial financial support.

\section{A. The HFE of the one-dimensional spinless fermionic Hubbard model up to order $\boldsymbol{\beta}^{6}$}

In reference [9] we calculated the $\beta$-expansion of the HFE of the normalized one-dimensional spin- $S$ of the $X X Z$ Heisenberg model with single-ion anisotropy term in the presence of a longitudinal magnetic field up to the order $\beta^{6}$, in terms of the rescaled spin operator $\vec{s}=\vec{S} / \sqrt{S(S+1)}$.

In the present work we have applied equation $\left[6\right.$ ) to equation (B) of reference [9], with $\|\overrightarrow{\mathbf{S}}\|=\frac{\sqrt{3}}{2}$, to derive the $\beta$-expansion, up to the order $\beta^{6}$, of the HFE of the one-dimensional fermionic spinless Hubbard model. We have obtained

$$
\begin{aligned}
W(t, V, \mu ; \beta)= & -\frac{\ln 2}{\beta}-\frac{1}{2} \mu+\frac{1}{4} V \\
& +\left(-\frac{1}{4} t^{2}+\frac{1}{4} V \mu-\frac{5}{32} V^{2}-\frac{1}{8} \mu^{2}\right) \beta \\
& +\left(\frac{1}{16} V \mu^{2}-\frac{1}{16} t^{2} V+\frac{1}{16} V^{3}-\frac{1}{8} V^{2} \mu\right) \beta^{2}
\end{aligned}
$$




$$
\begin{aligned}
+ & \left(-\frac{1}{48} V \mu^{3}+\frac{1}{16} t^{2} \mu^{2}+\frac{7}{96} t^{2} V^{2}+\frac{1}{64} V^{2} \mu^{2}+\frac{1}{96} V^{3} \mu\right. \\
& \left.-\frac{31}{3072} V^{4}+\frac{1}{32} t^{4}-\frac{1}{8} t^{2} V \mu+\frac{1}{192} \mu^{4}\right) \beta^{3} \\
+ & \left(-\frac{7}{256} t^{2} V^{3}-\frac{23}{384} V^{3} \mu^{2}-\frac{1}{128} V^{5}-\frac{1}{32} t^{2} V \mu^{2}+\frac{1}{32} t^{4} V\right. \\
& \left.+\frac{1}{24} V^{2} \mu^{3}+\frac{1}{16} t^{2} V^{2} \mu+\frac{7}{192} V^{4} \mu-\frac{1}{96} V \mu^{4}\right) \beta^{4} \\
+ & -\frac{47}{1536} t^{4} V^{2}-\frac{1}{96} t^{2} \mu^{4}-\frac{1}{32} t^{4} \mu^{2}-\frac{239}{7680} V^{5} \mu \\
& -\frac{1}{144} t^{6}+\frac{287}{36864} V^{6}-\frac{31}{1152} V^{3} \mu^{3}-\frac{1}{2880} \mu^{6} \\
& -\frac{21}{2560} t^{2} V^{4}+\frac{1}{480} V \mu^{5}+\frac{7}{192} t^{2} V^{3} \mu+\frac{5}{1536} V^{2} \mu^{4} \\
& \left.-\frac{23}{384} t^{2} V^{2} \mu^{2}+\frac{1}{24} V t^{2} \mu^{3}+\frac{139}{3072} V^{4} \mu^{2}+\frac{1}{16} V t^{4} \mu\right) \beta^{5} \\
+ & \frac{13}{768} V t^{2} \mu^{4}-\frac{1}{64} t^{4} V^{2} \mu+\frac{157}{1536} t^{2} V^{3} \mu^{2}-\frac{29}{10240} V^{7} \\
& +\frac{1}{128} V t^{4} \mu^{2}-\frac{13}{192} t^{2} V^{2} \mu^{3}-\frac{7}{576} V^{4} \mu^{3}-\frac{53}{768} t^{2} V^{4} \mu \\
& +\frac{17}{11520} V \mu^{6}+\frac{83}{23040} t^{4} V^{3}+\frac{1603}{92160} t^{2} V^{5}-\frac{17}{1920} V^{2} \mu^{5} \\
& -\frac{119}{30720} V^{5} \mu^{2}+\frac{389}{46080} V^{6} \mu-\frac{11}{768} V t^{6} \\
& \left.+\frac{41}{2304} V^{3} \mu^{4}\right) \beta^{6}+O\left(\beta^{7}\right) .
\end{aligned}
$$

\section{References}

1. Hubbard J., Proc. R. Soc. London, Ser. A, 1963, 276, 238; doi 10.1098/rspa.1963.0204

2. Hubbard J., Proc. R. Soc. London, Ser. A, 1964, 277, 237; doi 10.1098/rspa.1964.0019

3. Jördens R., Strohmaier N., Gunter K., Moritz H., Esslinger T., Nature, 2008, 455, 204; doi 10.1038/nature07244

4. Schneider U., Hackermuller L., Will S., Best Th., Bloch I., Costi T.A., Helmes R.W., Rasch D., Rosch A., Science, 2008, 322, 1520; doi 10.1126/science.1165449

5. Esslinger T., In: Annual Review of Condensed Matter Physics, Vol. 1, Langer J.S. (Ed.), Annual reviews, Palo Alto, CA, 2010, 129-152; doi 10.1146/annurev-conmatphys-070909-104059.

6. Simon J., Bakr W.S., Ma R., Tai M.E., Preiss Ph.M., Greiner M., Nature, 2011, 472, 307; doi 10.1038/nature09994

7. Verwey E.J.W., Haaymann P.W., Physica, 1941, 8, 979; doi 10.1016/S0031-8914(41)80005-6

8. Kobayashi K., Susaki T., Fujimori A., Tonogai T., Takagi H., Europhys. Lett., 2002, 59, 868; doi $10.1209 / \mathrm{epl} / \mathrm{i2002-00123-2}$

9. Rojas O., de Souza S.M., Corrêa Silva E.V., Thomaz M.T., Eur. Phys. J. B., 2005, 47, 165; doi 10.1140/epjb/e2005-00310-5

10. Sznajd J., Becker K., J. Phys.: Condens. Matter, 2005, 17, 7359; doi $10.1088 / 0953-8984 / 17 / 46 / 020$

11. Haldane F.D.M., Phys. Rev. Lett., 1980, 45, 1358; doi 10.1103/PhysRevLett.45.1358 


\title{
До симетрії частинка-дірка ферміонної безспінової моделі Габбарда в $D=1$
}

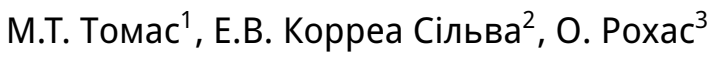 \\ ${ }^{1}$ Інститут фізики, Федеральний університет Флуміненсе, Нітерой-Рж, Бразилія \\ 2 Факультет математики, фізики та інформатики, технологічний факультет, Державний університет \\ Ріо-де-Жанейро, Ресенде-РЖ, Бразилія \\ 3 Факультет точних наук, Федеральний університет м. Лаврас, Лаврас-МЖ, Бразилія
}

\begin{abstract}
Ми наново переглядаємо симетрію частинка-дірка одновимірної $(D=1)$ ферміонної безспінової моделі Габбарда, пов'язуючи цю симетрію з інваріантністю вільної енергії Гельмгольца одновимірної спін-1/2 $X X Z$ моделі Гайзенберга, при інверсії (перекиданні) поздовжнього магнітного поля і при довільній скінченній температурі. В результаті порівняння двох режимів ланцюжкової моделі, коли число частинок в одному режимі дорівнює числу дірок в іншому, знайдено, що в загальному, їх термодинаміка є подібною, але не ідентичною: обидві моделі мають однакові функції питомої теплоємності та ентропії, але різні внутрішню енергію на вузол, кореляційні функції перших сусідів і число частинок на вузол. Завдяки цій симетрії, різниця між кореляційними функціями перших сусідів $\epsilon$ пропорційною до $z$-компоненти намагніченості $X X Z$ моделі Гайзенберга. Представлені в цій статті результати справедливі для довільного значення параметра сили взаємодії $V$, який описує притягальну/нульову/відштовхувальну взаємодію сусідніх ферміонів.
\end{abstract}

Ключові слова: квантово-статистична механіка, сильноскорельована електронна система, моделі спінових ланцюжків 